\title{
Non-Forward Balitsky-Kovchegov Equation and Vector Mesons
}

\author{
Robi Peschanski ${ }^{1}$, Cyrille Marquet ${ }^{2}$ and Gregory Soyez ${ }^{3}$ \\ 1- Service de Physique Théorique - CEA/Saclay, 91191 Gif-sur-Yvette Cedex, FRANCE \\ 2- RIKEN BNL Research Center, Brookhaven National Laboratory, Upton, NY 11973, USA \\ 3- Physics Department, Brookhaven National Laboratory, Upton, NY 11973, USA
}

\begin{abstract}
Considering the Balitsky-Kovchegov QCD evolution equation in full momentum space, we derive the travelling wave solutions expressing the nonlinear saturation constraints on the dipole scattering amplitude at non-zero momentum transfer. A phenomenological application to elastic vector meson production shows the compatibility of data with the QCD prediction: an enhanced saturation scale at intermediate momentum transfer.
\end{abstract}

\section{Motivation}

The saturation of parton densities at high energy has been mainly studied for the forward dipole-target scattering amplitude $\mathcal{T}(r, q=0, Y)$, where $r, q, Y$ are, respectively, the dipole size, the momentum transfer and the total rapidity of the process. For instance, the corresponding QCD Balitsky-Kovchegov (BK) equation [2] has been shown to provide a theoretical insight on the "geometric scaling" properties [3] of the related $\gamma^{*}$-proton cross-sections. Indeed, it can be related to the existence of a scaling for $\mathcal{T}(r, q=0, Y) \sim \mathcal{T}\left(r^{2} Q^{2}(Y)\right)$ where the saturation scale is $Q^{2}(Y) \sim \exp c Y$ and the constant $c$ can be interpreted as the critical speed of "travelling wave" solutions of the nonlinear BK equation [4]. Our theoretical and phenomenological subjects are the extension of these properties to the non-forward amplitude $\mathcal{T}(r, q \neq 0, Y)$, which is phenomenologically relevant for the elastic production of vector mesons in deep inelastic scattering.

\section{BK equation in full momentum space}

In order to study the properties of $\mathcal{T}(r, q \neq$ $0, Y)$, one has first to deal with both conceptual and technical difficulties. It is known that the BK formalism has been originally derived in impact parameter $b$ but then its validity especially at large $b$ is questionable, since it leads to non physical power-law tails. Hence we start with the formulation of the BK equation in momentum $q$, which is more local but has a non-trivial nonlinear form [5]. In fact, despite this problem, the general method of travelling wave solutions can be extended in the non-forward domain [6]. It consists in 3 steps: first, one

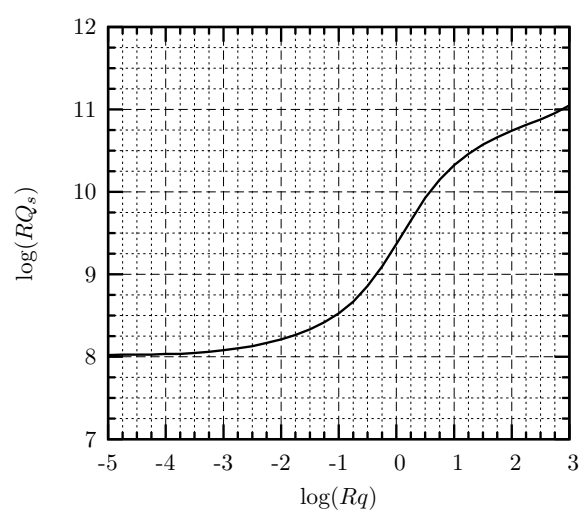

Figure 1: $q^{2}$-dependent saturation scale solves the equation restricted to its linear part which is related to the non-forward Balitsky Fadin Kuraev Lipatov (BFKL) equation 
[7] for the dipole-dipole amplitude via factorisation and whose solution takes the form of a linear superposition of waves. Second, one finds that the nonlinearities act by selecting the travelling wave with critical speed $c$, in a way which, interestingly, is independent of the specific structure of the nonlinear damping terms. Third, one obtains after enough rapidity evolution, a solution which appears independent from initial conditions $\left(\mathcal{T}_{0} \sim r^{2 \gamma_{0}}\right)$, provided these are sharper than the critical travelling wave front profile $\mathcal{T} \sim r^{2 \gamma_{c}}$, with $\gamma_{0}>\gamma_{c}$. Interestingly enough, QCD color transparency satisfies this criterium. Applying these gen-
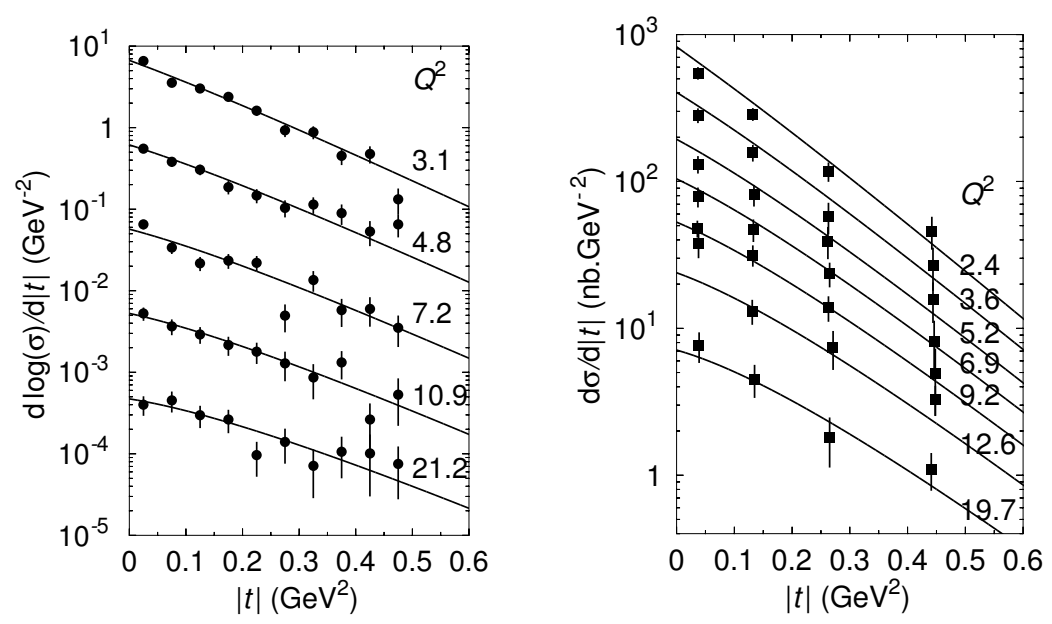

Figure 2: $\rho(\mathrm{H} 1)$ and $\phi$ (ZEUS) differential cross-sections at $W=75 \mathrm{GeV}$

eral results on the non-forward case one finds the following QCD predictions, depending on the relative magnitude of three scales involved in the process, namely $q, k_{T}^{-1}$ (the target size) and $k_{P}^{-1} \equiv r$ (the projectile i.e. dipole size).

- Near-Forward region $q \ll k_{T} \ll k_{P}: Q_{s}^{2}(Y) \sim k_{T}^{2} \exp c Y$

- Intermediate transfer region $k_{T} \ll q \ll k_{P}: Q_{s}^{2}(Y) \sim q^{2} \exp c Y$

- High transfer region $q \ll k_{T} \ll k_{P}$ : No saturation.

Our main prediction is thus the validity of the forward travelling wave solution extended in the non-forward intermediate-transfer domain but with an enhanced saturation scale by the ratio $q^{2} / k_{T}^{2}$, where $k_{T}$ is a typically small, nonperturbative scale. Hence we are led to predict geometric scaling properties with a purely perturbative initial saturation scale given by the transverse momentum. This saturation scale enhancement prediction is confirmed by numerical simulations of the BK solutions as shown in Fig.1.

\section{QCD Saturation Model for Exclusive VM production}

The differential cross-section for exclusive vector meson (VM) production at HERA, see Fig.2, can be theoretically obtained from the non-forward dipole-proton amplitude and from $\Phi_{T, L}^{\gamma^{*} V}$, the overlap functions between the (longitudinal and transverse) virtual photon and vector meson wave-functions [8]. For completion, we used two different VM wave-functions 
of the literature, without noticeable difference in our conclusions. One writes

$$
\frac{d \sigma_{T, L}^{\gamma^{*} p \rightarrow V p}}{d q^{2}}=\frac{1}{16 \pi}\left|\int d^{2} r \int_{0}^{1} d z \Phi_{T, L}^{\gamma^{*} V}\left(z, r ; Q^{2}, M_{V}^{2}\right) e^{-i z q \cdot r} \mathcal{T}(r, q, Y)\right|^{2},
$$

Following theoretical prescriptions, we consider a forward dipole-proton amplitude $\mathcal{N}_{\text {IIM }}$ satisfactorily describing the total DIS cross-sections in a saturation model [9]. We just make the saturation scale varying with $q^{2}$, following the trend shown in Fig.1 and starting from the forward model one $Q_{s}^{2}(Y)$, one writes

$$
T(r, q ; Y)=2 \pi R_{p}^{2} e^{-B q^{2}} \mathcal{N}_{I I M}\left(r^{2} Q_{s}^{2}(Y, q)\right) ; Q_{s}^{2}(q, Y)=Q_{s}^{2}(Y)\left(1+c q^{2}\right)
$$

\begin{tabular}{|c|c|c|}
\hline Cross-sections & $q^{2}$-Sat. & fixed-Sat. \\
\hline$\rho, \sigma_{\mathrm{el}}$ & 1.156 & 1.732 \\
\hline$\rho, \frac{d \sigma}{d t}$ & 1.382 & 1.489 \\
\hline$\phi, \sigma_{\mathrm{el}}$ & 1.322 & 2.247 \\
\hline$\phi, \frac{d \sigma}{d t}$ & 1.076 & 0.931 \\
\hline \hline Total & 1.212 & 1.480 \\
\hline \hline
\end{tabular}

Table 1: Comparison of the $\chi^{2} /$ points

The factor $2 \pi R_{p}^{2} e^{-B q^{2}}$ comes from the nonperturbative proton form factor. For clarity of the analysis, we considered only $B$ and $c$ as free parameters of the non-forward parametrisations, the others being independently fixed by the forward analysis.

In Table 1 , one displays the $\chi^{2} /$ point obtained by a fit of $\rho$ (47 data points) and $\phi$ (34 points) total elastic production crosssections and of $\rho$ (50 data points) and $\phi(70$ points) differential cross-sections. The Table compares the saturation fits for fixed and $q^{2}$-dependent scales, with a favour for the enhanced-scale model in the total. The model gives a comparable fit with a more conventional non-saturation model using a $Q^{2}$-dependent slope $B \propto M_{V}^{2}+Q^{2}$. Some of our results for the cross-sections are displayed in the figures. In Fig.2, one shows the results of the fit for $\rho$-production (H1) and $\phi$-production (ZEUS) differential cross-sections for a total $\gamma^{*}-p$ energy $W=75 \mathrm{GeV}$ and different $Q^{2}$ values. Let us finally present our predictions for the
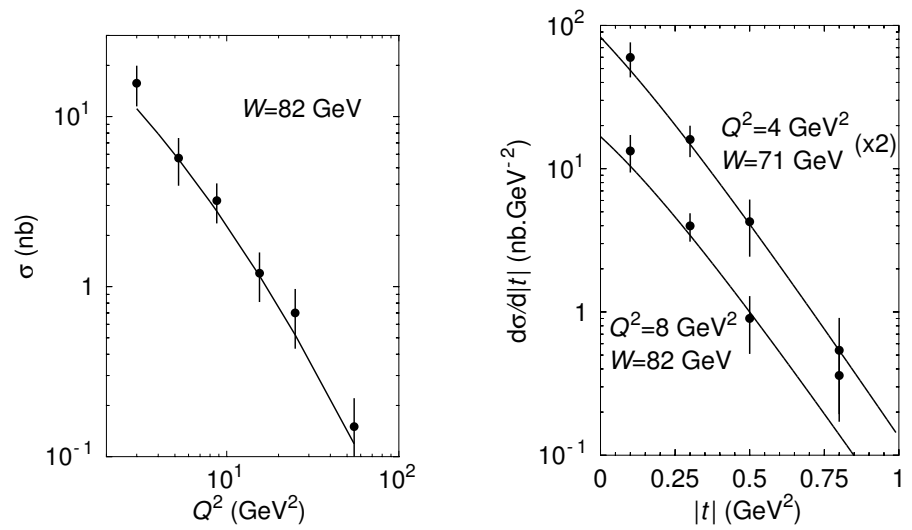

Figure 3: Predictions for the DVCS measurements. Left plot: cross-section, right plot: differential cross-section.

DVCS cross-section, which is obtained without any free parameter from our analysis. In 
Fig.3, they are compared with the available data and the agreement is good in the simple chosen parametrisation.

\section{Conclusions}

Let us summarize our new results

- Saturation at non-zero transfer: The Balitsky-Kovchegov QCD evolution equation involving full momentum transfer predicts (besides the known $q=0$ case) saturation in the intermediate transfer range, namely for $Q_{0}<q<Q$, where $Q_{0}$ (resp. $Q$ ) is the target (resp. projectile) typical scale.

- Characterisation of the universality class: The universality class of the corresponding travelling-wave solutions is governed by a purely perturbative saturation scale $Q_{s}(Y) \equiv$ $q^{2} \Omega(Y)$, where $\Omega(Y) \sim e^{c Y}$ is the same rapidity evolution factor as in the forward case. Consequently the intermediate transfer saturation scale gets enhanced by a factor $q^{2} / Q_{0}^{2}$.

- Phenomenology of Vector mesons: The QCD predictions are applied in the experimentally accessible intermediate transfer range of vector meson production. The model uses an interpolation between the forward and non-forward saturation scale together with a parameter-frozen forward saturation model. It fits better the data on $\rho$ (H1) and $\phi$ (ZEUS) cross-sections than for a non-enhanced saturation.

- Prospects: The next phenomenological prospect is to add charm to the discussion, both with the modification of the forward case by including the charm contribution [10] and by also considering the production of $\Psi$ mesons. On a theoretical ground, it would be interesting to go beyond the mean-field approximation of the BK equation.

\section{References}

[1] Slides: http: //indico. cern. ch/contributionDisplay. py? contribId=75\&sessionId $=7 \&$ conf Id $=9499$

[2] I. Balitsky, Nucl. Phys. B463 (1996) 99; Phys. Lett. B518 (2001) 235; Yu.V. Kovchegov, Phys. Rev. D60 (1999) 034008; Phys. Rev. D61 (2000) 074018.

[3] A.M. Stasto, K. Golec-Biernat and J. Kwiecinski, Phys. Rev. Lett. 86 (2001) 596; C. Marquet and L. Schoeffel, Phys. Lett. B639 (2006) 471.

[4] S. Munier and R. Peschanski, Phys. Rev. Lett. 91 (2003) 232001; Phys. Rev. D69 (2004) 034008; D70 (2004) 077503.

[5] C. Marquet and G. Soyez, Nucl. Phys. A760 (2005) 208.

[6] C. Marquet, R. Peschanski and G. Soyez, Nucl. Phys. A756 (2005) 399.

[7] L. N. Lipatov, Sov. Phys. JETP 63, 904 (1986) [Zh. Eksp. Teor. Fiz. 90, 1536 (1986)].

[8] C. Marquet, R. Peschanski and G. Soyez, Exclusive vector meson production at HERA from QCD with saturation, hep-ph/0702171.

[9] E. Iancu, K. Itakura and S. Munier, Phys. Lett. B590 (2004) 199.

[10] K. Golec-Biernat and S. Sapeta, Phys. Rev. D74 (2006) 054032;

H. Kowalski, L. Motyka and G. Watt, Phys. Rev. D74 (2006) 074016;

G. Soyez, Saturation QCD predictions with heavy quarks at HERA, arXiv.0705.3672. 